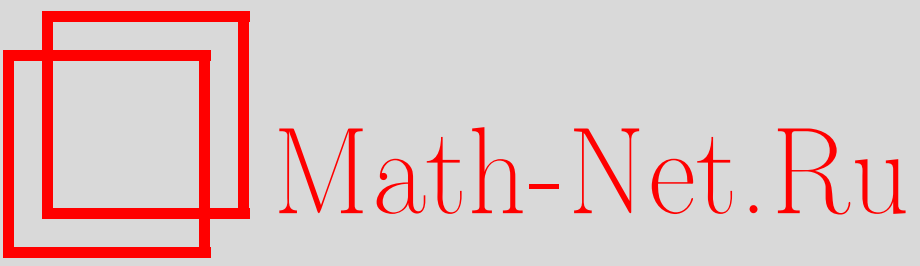

Г. В. Ефимов, О стабильности квантовой электродинамики и квантовой хромодинамики, ТМФ, 2004, том 141, номер 1, 80-99

DOI: https://doi.org/10.4213/tmf112

Использование Общероссийского математического портала Math-Net.Ru подразумевает, что вы прочитали и согласны с пользовательским соглашением

http://www.mathnet.ru/rus/agreement

Параметры загрузки:

IP: 18.234 .156 .22

26 апреля 2023 г., 15:45:59 
ТЕОРЕТИЧЕСКАЯ

И МАТЕМАТИЧЕСКАЯ

ФИЗИКА

Том 141, № 1

октябрь, 2004

(C) 2004 г.

Г.В. Ефимов*

\section{О СТАБИЛЬНОСТИ КВАНТОВОЙ ЭЛЕКТРОДИНАМИКИ И КВАНТОВОЙ ХРОМОДИНАМИКИ}

Рассматривается энергия вакуума в КЭД как в системе заряженных фермионов и бозонов и в КХД как в системе кварков (фермионов) и глюонов (бозонов) в самодуальном поле с постоянной напряженностью. Показано, что причиной неустойчивости является неустойчивость бозонов в самодуальном вакуумном поле. Для глобальной устойчивости системы, состоящей из фермионов и бозонов, необходимо, чтобы фермионов было "достаточно" много. Ненулевое самодуальное поле, ведущее к конфайнменту фермионов, реализует минимум энергии вакуума в случае, когда наименьшую массу в системе частиц имеет бозон. Поэтому конфайнмент не возникает в КЭД, где наименьшую массу в системе частиц имеет фермион - электрон, и возникает в КХД, где наименьшую массу имеет бозон - глюон.

Ключевые слова: квантовая электродинамика, квантовая хромодинамика, конфайнмент, самодуальное поле, энергия вакуума, устойчивость.

\section{1. ВВЕДЕНИЕ}

В настоящее время общепринято считать, что конфайнмент связан с возникновением кварк-кваркового линейно растушего потенциала или кварк-антикварковой "струны" и может быть объяснен только вне рамок теории возмущений (см., например, последний обзор на эту тему [1]). При этом проблема конфайнмента одного изолированного кварка вообще не ставится и, по всей видимости, не может быть поставлена. Попытки аналитического описания конфайнмента встречают отрицательное предубежденное отношение, и причина такого отношения, по нашему мнению, состоит в том, что до сих пор не найдено простое и удобное аналитическое представление для пропагаторов кварков и глюонов в области конфайнмента, которое сделало бы вычисления с ними не столь громоздкими (см., например, [2]).

Наша точка зрения состоит в том, что все явления адронной физики описываются в рамках квантовой теории поля. Это значит, что имеется КХД-лагранжиан кварков

* Лаборатория теоретической физики им. Н. Н. Боголюбова, Объединенный институт ядерных исследований, Дубна, Россия. E-mail: efimovg@thsun1.jinr.ru 
и глюонов, при этом эффективная константа связи должна быть малой и все расчеты можно проводить в рамках теории возмушений, включая образование связанных состояний, которые должны описываться уравнениями типа уравнения Бете-Солпитера. Основной вопрос заключается в том, какие пропагаторы должны быть использованы для описания кварков и глюонов в области конфайнмента. Ясно, что стандартные пропагаторы Дирака для кварков и Клейна-Гордона для глюонов, соответствующие плоским волнам, не дают правильного описания этих частиц в рассматриваемой области.

Мы считаем, что конфайнмент не связан с так называемым переходом к режиму сильной связи в КХД, т.е. конфайнмент не возникает в результате суммирования диаграмм теории возмущений.

Мы придерживаемся гипотезы, что самодуальное глюонное поле с постоянной напряженностью является хорошим кандидатом на роль вакуумного глюонного поля, поскольку оно удовлетворяет уравнениям Янга-Миллса и ведет к аналитическому конфайнменту, т.е. пропагаторы кварков в этом поле становятся целыми аналитическими функциями гауссовского типа в импульсном пространстве, и кварки не могут существовать как обычные частицы.

Следует особо отметить, что гауссовский характер поведения пропагаторов приводит к реджевскому поведению спектра адронов - мезонов и барионов. В частности, было показано [3], что основные черты адронного спектра можно объяснить, предположив, что пропагаторы кварков и глюонов являются просто гауссовскими экспонентами и адроны возникают как решения уравнения Бете-Солпитера. Была построена модель индуцированных кварковых токов (см. [4], [5]), основанная на предположении, что вакуум КХД реализуется самодуальным глюонным полем с постоянной напряженностью. Проведенные расчеты показали, что модель согласуется с имеюшимися экспериментальными данными.

Другим аргументом в пользу серьезного отношения к самодуальным полям с постоянной напряженностью является тот факт, что эти поля, будучи точными решениями уравнений Янга-Миллса, приводят к минимуму энергии глюонного вакуума при ненулевой напряженности поля [6], [7]. Однако самодуальное поле и в КЭД является решением уравнений Максвелла. Поэтому небезынтересно сравнить роль самодуального поля в КЭД и КХД и понять, в чем состоит принципиальная разница мира КЭД и мира КХД с точки зрения влияния самодуальных полей на стабильность систем частиц в этих двух мирах.

В работах [6], [7], посвященных вычислению энергии вакуума в самодуальном поле, рассматривалась система, состояшая только из глюонов. Нам такой подход представляется недостаточным, поскольку кварки и глюоны представляют единую систему и находятся в едином вакууме. Поэтому в КХД физическая система состоит из кварков и глюонов, а в КЭД - из всех заряженных частиц (фермионов и бозонов) и электромагнитного поля.

Наше предположение состоит в том, что вакуумное поле и константа связи должны быть полностью независимыми параметрами в лагранжианах КЭДи КХД. Это означа- 
ет, что при равенстве нулю одного из них зависимость от другого не должна исчезать из соответствуюшего лагранжиана. Обычно вакуумное поле рассматривается как внешнее поле, на практике это означает, что в лагранжиане производится замена $A \rightarrow A+B$, где $A$ и $B$ - внешнее и квантованное поля, затем рассматривается получившийся лагранжиан, в который внешнее поле входит в комбинации $e B$ и появляется кинетический вклад внешнего поля в энергию вакуума, пропорциональный $B^{2}$. Если же производится замена $A \rightarrow A+B / e$, то в лагранжиан внешнее поле войдет без множителя $e$, но кинетический вклад внешнего поля в энергию вакуума будет пропорционален $B^{2} / e^{2}$, и переход к пределу $e \rightarrow 0$ при отличном от нуля поле $B$ невозможен.

Мыпридерживаемся точки зрения, что если поле $B$ реализует вакуум КЭДили КХД, то это означает, что оно внутренне присуще рассматриваемой системе частиц, т.е. оно не является внешним полем в обычном понимании и изначально должно быть введено в лагранжиан системы полей так же, как вводится масса частиц. При этом вакуумное поле непосредственно не связано с зарядом и может быть отлично от нуля даже при равенстве нулю заряда $e=0$ или константы связи $g=0$, которые определяют силу взаимодействия частищ с фотонами или глюонами. Величина напряженности вакуумного поля должна определяться минимумом энергии системы частиц, находящихся в этом вакууме. Таким образом, вакуумное поле объединяет частишы даже в отсутствие квантового взаимодействия.

Лагранжиан системы частиц в вакуумном поле получим следуюшим образом. В исходном лагранжиане произведем замену $A \rightarrow A+B / e$, а затем опустим кинетический вклад внешнего поля в энергию вакуума, пропорциональный $B^{2} / e^{2}$. Выбор лагранжиана таким способом согласуется с общей формулировкой лагранжевой динамики, поскольку к лагранжиану всегда можно добавить полную производную, что не меняет динамические уравнения, описываюшие рассматриваемую систему полей.

Теперь о способе вычислений. Мы считаем, что гамильтонов подходпри вычислениях энергии вакуума не является наилучшим, поскольку, во-первых, гамильтониан в КТП не является хорошо определенным оператором (см., например, [8]) и, во-вторых, не ясно, имеем ли мы гамильтонову систему при включении самодуального поля, поскольку лагранжиан оказьвается явно зависящим от времени, а все вычисления проводятся в евклидовой метрике. Единственный результат, который с математической точки зрения достигнут в КТП, - это правила построения $S$-матрицы как унитарного оператора на пространстве Фока и производящего функционала $Z$ для функций Грина из заданного лагранжиана системы полей. При этом регуляризация, способ вычислений и перенормировочная процедура с правилом построения необходимых контрчленов для устранения расходящихся выражений играют определяющую роль. Последнее обстоятельство подтверждает то, что к гамильтонову методу в КТП надо относиться с большой осторожностью. Поэтому основным инструментом для наших вычислений будет производящий функционал.

Результаты, полученные в данной работе, можно сформулировать следующим образом. Показано, что как в КЭД, так и в КХД фермионы ведут к стабильности, а бозоны 
к нестабильности системы частиц в самодуальном поле с постоянной напряженностью.

В КЭД система заряженных фермионов и бозонов стабильна, т.е. минимум энергии вакуума реализуется при нулевом электромагнитном самодуальном поле с постоянной напряженностью, если, во-первых, число бозонов меньше удвоенного числа фермионов и, во-вторых, наилегчайшими частицами в системе являются фермионы. Именно эту ситуацию мы наблюдаем в Природе: практически все стабильные частицы (электрон и протон) являются фермионами, фермионов явно больше, чем бозонов, и легчайшей заряженной частицей является электрон, масса которого сушественно меньше масс остальных заряженных частиц. Таким образом, ненулевое самодуальное поле с постоянной напряженностью не может реализовать электромагнитный вакуум в имеющейся в Природе системе заряженных фермионов и бозонов.

Если же мы предположим, что существует гипотетический мир КЭД, состоящий из фермионов и бозонов, причем число фермионов больше числа бозонов, но массы бозонов меньше массы фермионов, то такой мир глобально стабилен, но вакуум КЭДв таком гипотетическом мире реализуется при ненулевом самодуальном поле. Наличие ненулевого поля ведет к возникновению конфайнмента заряженных фермионов и бозонов, и вместо заряженных частиц возникают нейтральные связанные состояния. Этот пример качественно правильно передает ситуацию в КХД, где роль заряженного бозона с малой массой играют самодействующие безмассовые глюоны.

В КХД в глюонном самодуальном поле с постоянной напряженностью кварк-глюонная система глобально стабильна, если число независимых кварков, т.е. кварков с различными ароматами, равно или больше двух. Напрашивается вывод, что именно это обстоятельство объясняет выделенность изотопической группы $S U_{f}(2)$ и практическое равенство масс $u$ - и $d$-кварков. Существование безмассового самодействующего бозона - глюона - приводит к тому, что возможен минимум энергии вакуума при конечной напряженности $\Lambda$ глюонного самодуального поля, величина которой определяется соотношением между хромодинамической шкалой $\Lambda_{\mathrm{KХ}}$ и и массами кварков $M_{f}$. Наличие ненулевого вакуумного самодуального поля ведет к аналитическому конфайнменту кварков и глюонов и возникновению стабильных состояний - адронов. Параметр $\Lambda$ определяет масштаб области конфайнмента и напрямую связан с наклоном траекторий Редже в спектре адронов.

Таким образом, результатом настоящей работы является следуюшее утверждение.

Причина конфайнмента - неустойчивость бозонов в самодуальном вакуумном поле. Для глобальной устойчивости системь, состоящей из фермионов и бозонов, необходимо, чтобъ фермионов было "достаточно" много. Конфайнмент не возникает в случае, когда наименьшую массу в системе частии имеет фермион (әлектрон в КЭД), и возникает, когда наименьшую массу имеет бозон (глюон в $K X$ Д). 


\section{2. КВАНТОВАЯ ЭЛЕКТРОДИНАМИКА}

Одним из решений уравнения Максвелла в пустоте $\partial_{\mu} F_{\mu \nu}(x)=0$ является самодуальное поле с постоянной напряженностью

$$
B_{\mu}(x)=\Lambda^{2} b_{\mu \nu} x_{\nu}, \quad B_{\mu \nu}=\partial_{\mu} B_{\nu}(x)-\partial_{\nu} B_{\mu}(x)=-2 \Lambda^{2} b_{\mu \nu},
$$

где $\Lambda$ характеризует напряженность самодуального поля и

$$
b_{\mu \nu}=-b_{\nu \mu}, \quad b_{\mu \rho} b_{\rho \nu}=-\delta_{\mu \nu}, \quad \tilde{b}_{\mu \nu}=\frac{1}{2} \epsilon_{\nu \mu \alpha \beta} b_{\alpha \beta}= \pm b_{\mu \nu} .
$$

Самодуальный или антисамодуальный тензор $b_{\mu \nu}$ характеризуется некоторым направлением

$\mathbf{n}_{j}=b_{j 0}$ в конфигурационном трехмерном пространстве.

Рассмотрим систему заряженных фермионов $\left\{\psi_{\mathrm{F}}\right\}$ и бозонов $\left\{\phi_{\mathrm{B}}\right\}$ с ненулевыми массами и одинаковым зарядом $e$. Наша конечная цель состоит в том, чтобы понять, как возникает конфайнмент в хромодинамике кварков и глюонов, поэтому для простоты фермионы и бозоны будем рассматривать как систему частиц с одинаковым зарядом.

Предположим, что самодуальное поле $B_{\mu}(x)$ реализует электромагнитньй вакуум. Согласно сказанному во введении лагранжиан системы $N_{\mathrm{F}}$ заряженных фермионов $\left\{\psi_{\mathrm{F}}\right\}$ и $N_{\mathrm{B}}$ заряженных бозонов $\left\{\phi_{\mathrm{B}}\right\}$ с ненулевыми массами и одинаковым зарядом $e$ постулируется в форме

$$
\mathcal{L}(x)=\mathcal{L}_{0}(x)+\mathcal{L}_{\mathrm{I}}(x),
$$

где свободный лагранжиан $\mathcal{L}_{0}(x)$ и лагранжиан взаимодействия $\mathcal{L}_{\mathrm{I}}(x)$ записываются как

$$
\begin{aligned}
\mathcal{L}_{0}(x)= & -\frac{1}{4}\left(\partial_{\mu} A_{\nu}(x)-\partial_{\nu} A_{\mu}(x)\right)^{2}+ \\
& +\sum_{\mathrm{F}}\left(\bar{\psi}_{\mathrm{F}}(x)\left[\gamma_{\mu} \nabla_{\mu}-M_{\mathrm{F}}\right] \psi_{\mathrm{F}}(x)\right)-\sum_{\mathrm{B}}\left(\phi_{\mathrm{B}}^{*}(x)\left[-\nabla^{2}+m_{\mathrm{B}}^{2}\right] \phi_{\mathrm{B}}(x)\right), \\
\mathcal{L}_{\mathrm{I}}(x)= & e \sum_{\mathrm{F}}\left(\bar{\psi}_{\mathrm{F}}(x) i \gamma_{\mu} \psi_{\mathrm{F}}(x)\right) A_{\mu}(x)- \\
& -i e\left[\left(\nabla_{\mu}(x) \phi(x)\right)^{*} \phi(x)-\phi^{*}(x) \nabla_{\mu}(x) \phi(x)\right] A_{\mu}(x)- \\
& -e^{2} \phi^{*}(x) \phi(x) A_{\mu}(x) A_{\mu}(x),
\end{aligned}
$$

где

$$
\begin{gathered}
\nabla_{\mu}(x)=\partial_{\mu}+i B_{\mu}(x)=\partial_{\mu}+i \Lambda^{2} b_{\mu \nu} x_{\nu}, \\
{\left[\nabla_{\mu}(x), \nabla_{\nu}(x)\right]=-2 i \Lambda^{2} b_{\mu \nu} .}
\end{gathered}
$$

Лагранжиан (2) инвариантен относительно калибровочных преобразований

$$
\begin{aligned}
A_{\mu}(x) & \rightarrow A_{\mu}(x)-\partial_{\mu} f(x), & B_{\mu}(x) & \rightarrow B_{\mu}(x), \\
\psi_{\mathrm{F}}(x) & \rightarrow e^{i e f(x)} \psi_{\mathrm{F}}(x), & \bar{\psi}_{\mathrm{F}}(x) & \rightarrow \bar{\psi}_{\mathrm{F}}(x) e^{-i e f(x),}, \\
\phi_{\mathrm{F}}(x) & \rightarrow e^{i e f(x)} \phi_{\mathrm{F}}(x), & \phi_{\mathrm{F}}^{*}(x) & \rightarrow \phi_{\mathrm{F}}^{*}(x) e^{-i e f(x)} .
\end{aligned}
$$


Поле $B_{\mu}(x)$ не меняется при калибровочных преобразованиях.

В лагранжиане (2) полностью разделены зависимости от параметров $\Lambda$ и $e$. Любой матричный элемент может быть представлен рядом

$$
A(e, \Lambda)=\sum_{n=n_{0}}^{\infty} e^{n} A_{n}(\Lambda) .
$$

Другими словами, если считать константу взаимодействия $e$ достаточно малой, то все вычисления можно проводить по теории возмушений, в то время как напряженность поля $\Lambda$ может принимать произвольные значения, при этом функции $A_{n}(\Lambda)$ могут быть точно вычислены. Таким образом, зависимость матричных элементов от напряженности поля $\Lambda$ можно изучать в каждом порядке теории возмушений по $e$.

Другое существенное замечание состоит в том, что самодуальное поле $B_{\mu}(x)$ не вносит никаких новых ультрафиолетовых (УФ) расходимостей в теорию, поэтому появление УФ-расходимостей связано только с разложением по заряду $е$ и имеет ту же структуру всех перенормировочных членов, что и при стандартном варианте перенормировки в КЭД. Кроме того, пропагаторы фермионов и бозонов в самодуальном поле $B_{\mu}(x)$ могут быть вычислены в явной форме. Таким образом, стандартные методы построения перенормированной $S$-матришы в КЭД полностью переносятся на данный случай.

Лагранжиан (2) описывает взаимодействие заряженных фермионов и бозонов, находящихся в вакуумном поле $B_{\mu}(x)$. Естественно возникает вопрос, какова напряженность $\Lambda$ этого поля? Поскольку мы предполагаем константу электромагнитного взаимодействия $\alpha=e^{2} /(4 \pi)$ малой, то напряженность $\Lambda$ должна быть выбрана из условия минимума энергии основного состояния - вакуума. Объектом нашего исследования будет производящий функционал

$$
\mathcal{Z}_{\mathrm{V}}=\iint \prod_{\mathrm{F}} D \psi_{\mathrm{F}} D \bar{\psi}_{\mathrm{F}} \prod_{\mathrm{B}} D \phi_{\mathrm{B}} D \phi_{\mathrm{B}}^{*} \int D A \exp \left(\int_{\mathrm{V}} d x \mathcal{L}(x)\right) .
$$

Нормировку функционального интеграла выбрем таким образом, что при $\Lambda=0$ и $e=0$ производяший функционал равен единице, $\mathcal{Z}_{\mathrm{V}}=1$.

Функциональный интеграл в (5) в низшем приближении теории возмушений при $e=0$ вычисляется в явном виде:

$$
\mathcal{Z}_{\mathrm{V}}^{(0)}=\prod_{\mathrm{F}} \operatorname{Det}\left[\frac{-\hat{\nabla}(x)+M_{\mathrm{F}}}{-\hat{\partial}+M_{\mathrm{F}}}\right] \prod_{\mathrm{B}} \operatorname{Det}\left[\frac{-\square+m_{\mathrm{B}}^{2}}{-\nabla^{2}+m_{\mathrm{B}}^{2}}\right] .
$$

Энергия вакуума определяется как

$$
E_{0}(\Lambda)=-\lim _{V \rightarrow \infty} \frac{1}{V} \ln \mathcal{Z}_{\mathrm{V}}^{(0)}=E_{\mathrm{F}}(\Lambda)+E_{\mathrm{B}}(\Lambda) .
$$

Представление (6) ясно показывает, что поведение функций $E_{\mathrm{F}}(\Lambda)$ и $E_{\mathrm{B}}(\Lambda)$ при $\Lambda \rightarrow \infty$ c необходимостью должно быть противоположного знака, т.е. если одна функция растет, то другая убывает. 
Воспользуемся формулами из приложения 1 и проведем перенормировку энергии вакуума условием

$$
\left.\frac{E_{\mathrm{vac}}(\Lambda)}{\Lambda^{4}}\right|_{\Lambda=0}=0
$$

В результате энергия вакуума системы заряженных фермионов и бозонов в КЭД имеет вид

$$
E_{\mathrm{vac}}(\Lambda)=\frac{4 \Lambda^{4}}{(4 \pi)^{2}}\left[\sum_{\mathrm{F}} 2 W\left(\frac{2 \Lambda^{2}}{M_{\mathrm{F}}^{2}}\right)-\sum_{\mathrm{B}} W\left(\frac{2 \Lambda^{2}}{m_{\mathrm{B}}^{2}}\right)\right] .
$$

Функция $W(X)$ определена формулой $(27)$ приложения 1.

Асимптотическое поведение энергии вакуума $E_{\mathrm{vac}}(\Lambda)$ при малых и больших $\Lambda$ задается формулами

$$
E_{\mathrm{vac}}(\Lambda)= \begin{cases}\frac{4 \Lambda^{4}}{(4 \pi)^{2}} \frac{1}{15}\left[\sum_{\mathrm{F}} 2\left(\frac{2 \Lambda^{2}}{M_{\mathrm{F}}^{2}}\right)^{2}-\sum_{\mathrm{B}}\left(\frac{2 \Lambda^{2}}{m_{\mathrm{B}}^{2}}\right)^{2}\right], \quad \Lambda \rightarrow 0 \\ \frac{4 \Lambda^{4}}{(4 \pi)^{2}} \frac{1}{3}\left[\sum_{\mathrm{F}} 2 \ln \left(\frac{2 \Lambda^{2}}{M_{\mathrm{F}}^{2}}\right)-\sum_{\mathrm{B}} \ln \left(\frac{2 \Lambda^{2}}{m_{\mathrm{B}}^{2}}\right)\right], \quad \Lambda \rightarrow \infty .\end{cases}
$$

На основании полученных формул для энергии вакуума (9) и (10) можно заключить, что в электромагнитном самодуальном поле с постоянной напряженностью:

1) система, состоящая только из заряженных фермионов, глобально стабильна;

2) система, состоящая только из заряженных бозонов, глобально нестабильна;

3) система заряженных фермионов и бозонов стабильна, если выполнены следуюшие условия:

а) число бозонов меньше удвоенного числа фермионов, $2 N_{\mathrm{F}}>N_{\mathrm{B}}$,

б) наилегчайшими частицами являются фермионы, так как должно выполняться неравенство

$$
\sum_{\mathrm{F}} \frac{2}{M_{\mathrm{F}}^{2}}>\sum_{\mathrm{B}} \frac{1}{m_{\mathrm{B}}^{2}}
$$

в этом случае минимум энергии вакуума реализуется при $\Lambda=0$.

Именно эту ситуацию мы наблюдаем в Природе: основные стабильные частицы являются фермионами (электрон и протон), фермионов явно больше, чем бозонов, и легчайшей заряженной частицей является электрон, масса которого сушественно меньше остальных заряженных частиц. Таким образом, ненулевое самодуальное поле с постоянной напряженностью не может реализовать электромагнитный вакуум в системе заряженных фермионов и бозонов, имеющихся в Природе.

Гипотетический мир КЭД. Для того чтобы лучше понять условие стабильности, рассмотрим гипотетический мир, состоящий из двух сортов заряженных частищ: фермионов с массой $M$ и бозонов с массой $m$, причем масса бозона меньше массы фермиона. Тогда энергию вакуума в самодуальном поле (9) с постоянной напряженностью можно записать в виде

$$
E_{\mathrm{vac}}(\Lambda) \approx \frac{4 \Lambda^{4}}{6(4 \pi)^{2}}\left[2 \ln \left(1+\frac{2}{5} \frac{2 \Lambda^{2}}{M_{\mathrm{F}}^{2}}\right)-\ln \left(1+\frac{2}{5} \frac{2 \Lambda^{2}}{m_{\mathrm{B}}^{2}}\right)\right],
$$


где мы для простоты использовали приближенное выражение (28) из приложения 1 для функции $W(X)$.

Наш гипотетический мир глобально стабилен, поскольку энергия вакуума растет при $\Lambda \rightarrow \infty$. Однако если масса бозона мала, $m_{\mathrm{B}}^{2}<M_{\mathrm{F}}^{2} / 2$, то функция $E_{\mathrm{vac}}(\Lambda)$ имеет отрицательный минимум при конечных $\Lambda>0$. Таким образом, вакуум КЭД в нашем гипотетическом мире реализуется при ненулевом самодуальном поле. Наличие ненулевого поля $B_{\mu}(x)$ ведет к возникновению конфайнмента заряженных фермионов и бозонов, поскольку их пропагаторы становятся целыми аналитическими функциями в импульсном пространстве. Вместо заряженных частиц возникают нейтральные связанные состояния, массы которых можно вычислить, используя соответствующие уравнения Бете-Солпитера.

Этот пример качественно правильно передает ситуацию в КХД, где роль заряженного бозона с малой массой играют самодействующие безмассовые глюоны.

\section{3. КВАНТОВАЯ ХРОМОДИНАМИКА}

КХД описывает кварки и глюоны, динамическое взаимодействие которых определяется лагранжианом

$$
\mathcal{L}(x)=-\frac{1}{2} \operatorname{Tr} \breve{G}_{\mu \nu}(x) \breve{G}_{\mu \nu}(x)+\sum_{f}\left(\bar{q}_{f}(x)\left[\gamma_{\mu}\left(\partial_{\mu}-i g \breve{A}_{\mu}(x)\right)-M_{f}\right] q_{f}(x)\right),
$$

где

$$
\begin{aligned}
\breve{G}_{\mu \nu}(x) & =\breve{F}_{\mu \nu}(x)-i g\left[\breve{A}_{\mu}(x), \breve{A}_{\nu}(x)\right], \\
\breve{F}_{\mu \nu}(x) & =\partial_{\mu} \breve{A}_{\nu}(x)-\partial_{\mu} \breve{A}_{\nu}(x), \quad \breve{A}_{\mu}(x)=A_{\mu}^{a}(x) t^{a},
\end{aligned}
$$

$t^{a}=\lambda^{a} / 2-$ матрицы цветовой группы $S U_{\mathrm{c}}(3)$.

Лагранжиан (12) инвариантен относительно калибровочных преобразований цветовой группы $S U_{\mathrm{c}}(3)$

$$
\begin{aligned}
q_{f}(x) & \rightarrow U(x) q_{f}(x), \quad \bar{q}_{f}(x) \rightarrow \bar{q}_{f}(x) U^{+}(x), \\
\breve{A}_{\mu}(x) & \rightarrow U(x) \breve{A}_{\mu}(x) U^{+}(x)-\frac{i}{g} U_{\mu}(x) U^{+}(x), \quad U_{\mu}(x)=\partial_{\mu} U(x), \\
\breve{G}_{\mu \nu}(x) & \rightarrow U(x) \breve{G}_{\mu \nu}(x) U^{+}(x) .
\end{aligned}
$$

Под самодуальным глюонным полем с постоянной напряженностью понимается поле $\breve{B}_{\mu}(x)($ см. [7]), которое при калибровочных преобразованиях преобразуется как

$$
\breve{B}_{\mu}(x) \rightarrow U(x) \breve{B}_{\mu}(x) U^{+}(x),
$$

и сушествует такая калибровка, что $\breve{B}_{\mu}(x)=\breve{n} B_{\mu}(x), \breve{n}=t^{a} n^{a}$, где $\breve{n}=$ const и поле $B_{\mu}(x)$ определено в (1). Как и в КЭД, самодуальный или антисамодуальный тензор 
$b_{\mu \nu}$ характеризуется некоторым направлением $\mathbf{n}$ в конфигурационном трехмерном пространстве, так что $b_{j 0}=\mathbf{n}_{j}$ и $b_{i j}=\varepsilon_{i j k} \mathbf{n}_{k}$. Постоянный вектор $n^{a}$ характеризуется двумя инвариантными параметрами $\left(n^{a} n^{a}\right)=1$ и $\left(n^{a} n^{b} n^{c} d^{a b c}\right)$ и определяет некоторое направление в цветовом пространстве $S U_{c}(3)$ (см. приложение 2 ). Калибровочное преобразование (14) означает врашение вектора $n^{a}$ в цветовом пространстве. Параметр $\Lambda$ характеризует напряженность самодуального поля. Поле $\breve{B}_{\mu}(x)$ является решением уравнений Янга-Миллса в пустоте.

Как и в случае КЭД, мы предполагаем, что самодуальное поле $\breve{B}_{\mu}(x)$ реализует хромодинамический вакуум.

Лагранжиан кварк-глюонной системы (12) в вакуумном самодуальном поле получается, если в лагранжиане (12) провести замену

$$
\breve{A}_{\mu}(x) \rightarrow \breve{A}_{\mu}(x)+\frac{1}{g} \breve{B}_{\mu}(x)
$$

и удалить постоянный кинетический член $\sim \Lambda^{4} / g^{2}$. Полученный лагранжиан инвариантен относительно калибровочных преобразований цветовой группы (13) и преобразования (14).

Выделим “свободную” часть лагранжиана (12) при $g=0$ :

$$
\mathcal{L}_{0}(x)=\sum_{f} \mathcal{L}_{f 0}(x)+\mathcal{L}_{q 0}(x) .
$$

Здесь лагранжиан для кварка с массой $M_{f}$ записывается как

$$
\mathcal{L}_{f 0}(x)=\left(\bar{q}_{f}(x)\left[\gamma_{\mu} \breve{\nabla}_{\mu}(x)-M_{f}\right] q_{f}(x)\right)
$$

где

$$
\begin{gathered}
\breve{\nabla}_{\mu}(x)=\partial_{\mu}+i \breve{B}_{\mu}(x)=\partial_{\mu}+i \Lambda^{2} \breve{n} b_{\mu \nu} x_{\nu}, \\
{\left[\breve{\nabla}_{\mu}(x), \breve{\nabla}_{\nu}(x)\right]=-2 i \Lambda^{2} \breve{n} b_{\mu \nu}, \quad \breve{n}=n^{a} t^{a} .}
\end{gathered}
$$

Квадратичная часть глюонного лагранжиана после некоторых преобразований с учетом калибровки $\left(\check{\nabla}_{\mu} A(x)_{\mu}(x)\right)=0$ или $\left(\check{\nabla}_{\mu}\right)^{a b}(x) A_{\mu}^{b}(x)=0$ приобретает вид

$$
\begin{aligned}
\mathcal{L}_{g 0}(x) & =-\frac{1}{2} \operatorname{Tr}\left[\breve{F}_{\mu \nu}(x) \breve{F}_{\mu \nu}(x)+2 \breve{B}_{\mu \nu} \breve{A}_{\mu}(x) \breve{A}_{\nu}(x)\right]= \\
& =-\frac{1}{2}\left(A_{\mu}(x)\left[-\check{\nabla}^{2}(x) \delta_{\mu \nu}+4 i \Lambda^{2} \check{n} b_{\mu \nu}\right] A_{\nu}(x)\right) .
\end{aligned}
$$

Мы не будем здесь выписывать в явном виде лагранжиан взаимодействия.

В случае КХД остаются справедливыми замечания, сделанные для КЭД. Повторим их.

Во-первых, самодуальное поле $\breve{B}_{\mu}(x)$ не вносит никаких новых УФ-расходимостей в теорию, поэтому появление УФ-расходимостей связано только с разложением по заряду $g$ и имеет ту же структуру всех перенормировочных членов, что и при стандартном 
варианте перенормировки в КХД. Кроме того, пропагаторы кварков и глюонов в самодуальном поле $\breve{B}_{\mu}(x)$ могут быть вычислены в явном виде. Таким образом, стандартные методы построения перенормированной $S$-матрицы в КХД полностью переносятся на данный случай.

Во-вторых, в лагранжиане (15) полностью разделены зависимости от параметров $g$ и $\Lambda$ в любых матричных элементах, т.е. для любого матричного элемента мы получаем выражения вида

$$
A(g, \Lambda)=\sum_{n=n_{0}}^{\infty} g^{n} A_{n}(\Lambda)
$$

Другими словами, если считать константу взаимодействия $g$ достаточно малой, все вычисления можно проводить в рамках теории возмущений, в то время как напряженность поля $\Lambda$ может принимать произвольные значения, при этом функции $A_{n}(\Lambda)$ могут быть точно вычислены. Таким образом, зависимость матричных элементов от напряженности поля $\Lambda$ можно изучать в каждом порядке теории возмушений по $g$.

Преж де чем перейти к вычислениям, нам представляется интересным сделать качественное предсказание поведения энергии вакуума в самодуальном поле с постоянной напряженностью в КХД исходя из полученного результата в КЭД. Итак, какие выводы можно сделать из формул (9) и (10)? Прежде всего, вклад кварков в энергию вакуума качественно совпадает с вкладом электронов в КЭД, т.е. кварки обеспечивают стабильность квантовой системы. В КХД “бозонов" нет, их роль играют глюоны благодаря их самодействию, чего не было у фотонов в КЭД. Поскольку глюоны яВляются бозонами, качественное поведение глюонного вклада в энергию вакуума описывается формулами, близкими к бозонному вкладу в КЭД с учетом нулевой массы глюонов. Эта зависимость при больших $\Lambda$ должна быть вида $\sim-\Lambda^{4} \ln \Lambda^{2}$, другими словами, глюоны ведут к нестабильности вакуума КХД. Следует обратить особое внимание на отрицательный знак этого вклада, в противоположность имеющимся в литературе формулам (см. [7]). Поскольку поведение при больших $\Lambda$ и у кваркового, и у глюонного вкладов в вакуумную энергию функционально одинаково $\left(\sim \Lambda^{4} \ln \Lambda^{2}\right)$, то различных типов кварков должно быть "достаточно много", чтобы обеспечить глобальную стабильность кварк-глюонной системы. Ниже мы вычислим необходимое число кварков.

Итак, в КХД, в отличие от КЭД, глюон играет роль легчайшего бозона (см. (10)). При малых $\Lambda$ имеется как положительный вклад от кварков, так и отрицательный вклад от глюонов. Только аккуратное вычисление должно показать, реализуется ли минимум энергии вакуума при ненулевых $\Lambda$. Если вакуум имеет минимум при $\Lambda=0$, т.е. самодуальное поле отсутствует, то это означает, что кварки и глюоны сушествуют как обычные частишы и описываются свободными уравнениями. Если же вакуум имеет минимум при ненулевых $\Lambda$, то это означает, что самодуальное глюонное поле с постоянной напряженностью реализует вакуум кварк-глюонной системы, кварки и глюоны находятся в этом поле и возникает конфайнмент кварков, т.е. реализуется так называемый аналитический конфайнмент. K анализу этой ситуации мы сейчас и перейдем. 
Объектом нашего исследования будет производяший функционал в низшем порядке теории возмущений при $g=0$

$$
\mathcal{Z}_{\mathrm{V}}^{(0)}=\int \prod_{f} D \psi_{f} D \bar{\psi}_{f} \int D A \delta(\nabla A) \Delta(A) \exp \left(\int_{\mathrm{V}} d x \mathcal{L}_{0}(x)\right)=\mathcal{Z}_{\mathrm{Vq}}^{(0)} \cdot \mathcal{Z}_{\mathrm{Vg}}^{(0)}
$$

Нормировка функционального интеграла выбрана таким образом, что при $\Lambda=0$ производящий функционал равен единице, $\mathcal{Z}_{\mathrm{V}}^{(0)}=1$.

Функциональные интегралы могут быть вычислены (см. приложения 5 и 6):

$$
\begin{aligned}
\mathcal{Z}_{\mathrm{Vq}}^{(0)} & =\int d \sigma_{\mathrm{q}} \exp \left(\int_{\mathrm{V}} d x \mathcal{L}_{\mathrm{q}}(x)\right)=\prod_{f} \operatorname{Det}\left[\frac{\gamma_{\mu} \breve{\nabla}_{\mu}(x)-M_{f}}{\gamma_{\mu} \partial_{\mu}-M_{f}}\right] \\
\mathcal{Z}_{\mathrm{Vg}}^{(0)} & =\frac{1}{N_{A}} \int D A \delta(\nabla A) \Delta(A) \exp \left(-\frac{1}{2} \int_{\mathrm{V}} d x\left(A_{\mu}\left[-\check{\nabla}^{2} \delta_{\mu \nu}+4 i \Lambda^{2} \check{n} b_{\mu \nu}\right] A_{\nu}\right)\right)= \\
& =\operatorname{Det}\left[\frac{-\square}{-\nabla^{2}+4 \Lambda_{\mathrm{g}}^{2}}\right] \operatorname{Det}\left[\frac{-\square}{-\nabla^{2}-4 \Lambda_{\mathrm{g}}^{2}}\right] .
\end{aligned}
$$

Соответственно для энергии вакуума имеем

$$
E_{0}(\Lambda)=E_{\mathrm{q}}(\Lambda)+E_{\mathrm{g}}(\Lambda)
$$

Качественно очевидно, что при больших $\Lambda$ поведение и кваркового, и глюонного вкладов в вакуумную энергию должно быть функционально одинаково, но противоположных знаков.

Кварковый вклад в энергию вакуума после перенормировки (8) равен (см. приложение 5)

$$
E_{\mathrm{q}}(\Lambda)=\frac{4 \Lambda^{4}}{(4 \pi)^{2}} W_{\mathrm{q}}\left(\frac{2 \Lambda^{2}}{M^{2}}\right)
$$

функция $W_{\mathrm{q}}(X)$ определена в приложении 5. Функция $W(X)$ определена в $(27)$.

Глюонный вклад в энергию вакуума определен в приложении 6 (см. формулу (41)). Неопределенный параметр $H$ возникает как результат перенормировки глюонного вклада в энергию вакуума, в котором отсутствует какая-либо шкала масс. При этом подчеркнем, что в нашем подходе эта перенормировка напрямую не связана с перенормировкой константы связи $g$. Тем не менее параметр $H$ должен быть как-то связан с хромодинамической энергетической шкалой $\Lambda_{\mathrm{KХ} \text { д. }}$

Подставив формулы для $E_{\mathrm{q}}(\Lambda)$ и $E_{\mathrm{g}}(\Lambda)$ в $(21)$, получим

$$
E_{\mathrm{vac}}(\Lambda)=\frac{4 \Lambda^{4}}{(4 \pi)^{2}}\left[-\frac{1}{2} \ln \left(\frac{2 \Lambda^{2}}{H^{2}}\right)+\sum_{f} W_{\mathrm{q}}\left(\frac{2 \Lambda^{2}}{M_{f}^{2}}\right)\right] .
$$

Асимптотическое поведение энергии вакуума при больших $\Lambda$ имеет вид

$$
\mathcal{E}_{\mathrm{vac}}(\Lambda) \rightarrow\left(-\frac{1}{2}+\frac{1}{3} N_{\mathrm{q}}\right) \frac{\Lambda^{4} \ln \Lambda^{2}}{(2 \pi)^{2}}
$$


где $N_{\mathrm{q}}=\sum_{f}-$ число кварков.

На основании полученных формул для энергии вакуума (23) и (24) можно сделать следующие заключения.

1. В глюонном самодуальном поле с постоянной напряженностью кварк-глюонная система глобально стабильна, если число независимых кварков не меньше двух, $N_{\mathrm{q}} \geqslant 2$. Возможно, это обстоятельство объясняет выделенность изотопической группы $S U_{f}(2)$ и практическое равенство масс $u$ - и $d$-кварков.

2. Минимум энергии вакуума в (23) определяется соотношением между напряженностью самодуального поля $\Lambda$, постоянной $H$, связанной с хромодинамической шкалой $\Lambda_{\mathrm{Kх}}$, и массами кварков $M_{f}$. При определенных соотношениях между этими параметрами возможен минимум энергии вакуума при ненулевых значениях $\Lambda$, что ведет к конфайнменту кварков в КХД. Параметр $\Lambda$ определяет масштаб области конфайнмента и напрямую связан с наклоном траекторий Редже в спектре адронов.

Следуюшей задачей является согласование параметров с основными экспериментальными данными: наклон траекторий Редже, массы адронов, ширины распадов и другие характеристики адронной физики.

ПРИЛОЖКНИЕ 1

\section{Функция Грина}

Наши вычисления основаны на следуюших формулах:

$$
\begin{aligned}
G(x, \Lambda, v) & =\frac{1}{-\square+\Lambda^{4} x^{2}+v} \delta(x)= \\
& =\frac{1}{(4 \pi)^{2}} \int_{s_{0}}^{\infty} \frac{d s}{s^{2}}\left(\frac{2 \Lambda^{2} s}{\operatorname{sh}\left(2 \Lambda^{2} s\right)}\right)^{2} e^{-v s-\Lambda^{2} x^{2} /\left(2 \operatorname{th}\left(2 \Lambda^{2} s\right)\right)} .
\end{aligned}
$$

Параметр $s_{0}$ определяет УФ-регуляризацию по “собственному времени”.

Пусть $A, B>-4 \Lambda^{2}$, тогда

$$
\begin{aligned}
W_{s_{0}}(\Lambda, A, B) & =\frac{1}{V} \operatorname{Tr} \ln \left[\frac{-\square+\Lambda^{4} x^{2}+A}{-\square+B}\right]= \\
& =\frac{4 \Lambda^{4}}{(4 \pi)^{2}} \int_{s_{0}}^{\infty} \frac{d s}{s}\left[\frac{e^{-B s}}{\left(2 \Lambda^{2} s\right)^{2}}-\frac{e^{-A s}}{\operatorname{sh}^{2}\left(2 \Lambda^{2} s\right)}\right] .
\end{aligned}
$$

При $A=B=M^{2}$

$$
W_{s_{0}}(\Lambda, M)=4 \Lambda^{4}\left[-\frac{1}{(4 \pi)^{2}} W\left(\frac{2 \Lambda^{2}}{M^{2}}\right)+\frac{1}{3} Z(M)\right] .
$$

Здесь

$$
\begin{aligned}
W(X) & =\int_{0}^{\infty} \frac{d s}{s} e^{-s}\left[\frac{1}{3}-\frac{1}{(X s)^{2}}+\frac{1}{\operatorname{sh}^{2}(X s)}\right]= \\
& = \begin{cases}\frac{1}{15} X^{2}+O\left(X^{3}\right), & X \rightarrow 0, \\
\frac{1}{3} \ln X+O(1), & X \rightarrow \infty .\end{cases}
\end{aligned}
$$


Для качественных рассуждений удобно ввести приближенную функцию

$$
W(X) \approx W_{a}(X)=\frac{1}{6} \ln \left(1+\frac{2}{5} X^{2}\right),
$$

имеюшую то же самое асимптотическое поведение при малых и больших $X$, что и $W(X)$.

УФ-расходимость параметризована константой

$$
Z(A)=\frac{1}{(4 \pi)^{2}} \int_{s_{0}}^{\infty} \frac{d s}{s} e^{-A s}
$$

определяющей нормировку регуляризованного выражения $W(0)=0$.

Фермионная энергия вакуума. Вычислим фермионную часть энергии вакуума:

$$
E_{\mathrm{F}}(\Lambda, M)=-\lim _{V \rightarrow \infty} \frac{1}{V} \operatorname{Tr} \ln \left[\frac{-\widehat{\nabla}(x)+M}{-\hat{\partial}+M}\right]=-\lim _{V \rightarrow \infty} \frac{1}{2 V} \operatorname{Tr} \ln \left[\frac{-\widehat{\nabla}^{2}(x)+M^{2}}{-\square+M^{2}}\right] .
$$

В представлении

$$
\widehat{\nabla}^{2}(y)=\nabla^{2}(y)-4 \Lambda^{2} \mathcal{P}
$$

матрица $\mathcal{P}$ обладает свойствами

$$
\begin{aligned}
\mathcal{P} & =\frac{i}{4}(\gamma b \gamma)=\frac{i}{4}\left(\gamma_{\mu} b_{\mu \nu} \gamma_{\nu}\right), \quad \gamma_{\mu} \gamma_{\nu}+\gamma_{\nu} \gamma_{\mu}=2 \delta_{\mu \nu}, \\
\mathcal{P}^{2} & =\frac{1}{2}\left(1 \mp \gamma_{5}\right), \quad \mathcal{P}^{3}=\mathcal{P}, \quad \operatorname{Tr} \mathcal{P}=0, \quad \operatorname{Tr} \mathcal{P}^{2}=2 .
\end{aligned}
$$

Удобно ввести проекционные операторы

$$
\begin{gathered}
\mathcal{R}_{(0)}=I-\mathcal{P}^{2}, \quad \mathcal{R}_{( \pm)}=\frac{1}{2}\left(\mathcal{P}^{2} \pm \mathcal{P}\right), \\
\mathcal{R}_{(j)} \mathcal{R}_{\left(j^{\prime}\right)}=\delta_{j j^{\prime}} \mathcal{R}_{(j)}, \quad j, j^{\prime}=-, 0,+, \\
\operatorname{tr} \mathcal{R}_{(0)}=2, \quad \operatorname{tr} \mathcal{R}_{( \pm)}=1 .
\end{gathered}
$$

Разложив оператор (29) по этим проекционным операторам, легко получить

$$
\begin{aligned}
\frac{1}{2} \operatorname{Tr} \ln & {\left[\frac{-\widehat{\nabla}^{2}+M^{2}}{-\square+M^{2}}\right]=\operatorname{Tr} \ln \left[\frac{-\square+\Lambda^{4} y^{2}+M^{2}}{-\square+M^{2}}\right]+} \\
& +\frac{1}{2} \operatorname{Tr} \ln \left[\frac{-\square+\Lambda^{4} y^{2}+M^{2}+4 \Lambda^{2}}{-\square+M^{2}}\right]+\frac{1}{2} \operatorname{Tr} \ln \left[\frac{-\square+\Lambda^{4} y^{2}+M^{2}-4 \Lambda^{2}}{-\square+M^{2}}\right]
\end{aligned}
$$

Воспользовавшись формулой (26), получим после простых преобразований

$$
E_{\mathrm{F}}(\Lambda, M)=4 \Lambda^{4}\left\{\frac{2}{(4 \pi)^{2}} W\left(\frac{2 \Lambda^{2}}{M^{2}}\right)+\frac{4}{3} Z(M)\right\} .
$$

Функция $W(X)$ определена формулой $(27)$.

Бозонная энергия вакуума. Вклад в энергию вакуума заряженного бозона массы $m$ равен

$$
\begin{aligned}
E_{\mathrm{B}}(\Lambda, m) & =\lim _{V \rightarrow \infty} \frac{1}{V} \operatorname{Tr} \ln \left[\frac{-\nabla^{2}+m^{2}}{-\square+m^{2}}\right]=\lim _{V \rightarrow \infty} \frac{1}{V} \operatorname{Tr} \ln \left[\frac{-\square+\Lambda^{2} x^{2}+m^{2}}{-\square+m^{2}}\right]= \\
& =4 \Lambda^{4}\left\{-\frac{1}{(4 \pi)^{2}} W\left(\frac{2 \Lambda^{2}}{m^{2}}\right)+\frac{1}{3} Z(m)\right\} .
\end{aligned}
$$

Используемый нами метод вычислений проше вычислений Швингера [9]. 


\section{Матрицы $\breve{n}$ и $\check{n}$}

ПРИЛОЖКЕНИЕ 2

Пусть в группе $S U_{\mathrm{c}}(3)$ задан некоторый постоянный вектор $n^{a}$. Он характеризуется двумя групповыми инвариантами

$$
(n n)=\left(n^{a} n^{a}\right)=1, \quad(\text { nnnd })=\left(n^{a} n^{b} n^{c} d^{a b c}\right) .
$$

Сушествует система координат, где этот вектор имеет вид

$$
n^{a}=\delta^{a 3} n^{3}+\delta^{a 8} n^{8}=\delta^{a 3} \sin \theta+\delta^{a 8} \cos \theta
$$

$$
(n n n d)=\left(n^{a} n^{b} n^{c} d^{a b c}\right)=\frac{1}{\sqrt{3}} \sin ^{2} \theta(3 \cos \theta-\sin \theta) .
$$

Вообще говоря, угол $\theta$ должен определяться условием минимума вакуумной энергии, однако мы поступим проше - выберем его равным нулю, т.е. $n^{a}=\delta^{a 8}$.

В кварковый лагранжиан входит постоянная $(3 \times 3)$-матрица

$$
\breve{n}=(n t)=t^{8}=\operatorname{diag}\left(\frac{1}{2 \sqrt{3}}, \frac{1}{2 \sqrt{3}},-\frac{1}{\sqrt{3}}\right) .
$$

В глюонньй лагранжиан входит постоянная $(8 \times 8)$-матрица в присоединенном представлении, которая эрмитова и в диагональной форме имеет вид

$$
\check{n}=\frac{\sqrt{3}}{4} \operatorname{diag}(1,1,-1,-1,0,0,0,0) .
$$

Производная $\nabla_{\mu}(x)$

ПРИЛОЖЕНИЕ 3

Рассмотрим оператор

$$
\nabla_{\mu}(x)=\partial_{\mu}+i \Lambda_{\mathrm{g}}^{2} b_{\mu \nu} x_{\nu}, \quad\left[\nabla_{\mu}, \nabla_{\nu}\right]=-2 i \Lambda_{\mathrm{g}}^{2} b_{\mu \nu}, \quad \Lambda_{\mathrm{g}}=\frac{\sqrt{3}}{4} \Lambda^{2} .
$$

Справедливы следуюшие соотношения:

$$
\begin{aligned}
& {\left[\nabla_{\mu}, \nabla^{2}\right]=-4 i \Lambda_{\mathrm{g}}^{2} b_{\mu \nu} \nabla_{\nu}, \quad \nabla_{\mu} b_{\mu \nu} \nabla_{\nu}=-4 i \Lambda_{\mathrm{g}}^{2},} \\
& \nabla_{\mu} \nabla^{2} \nabla_{\mu}=\left(\nabla^{2}\right)^{2}+\left(4 \Lambda_{\mathrm{g}}^{2}\right)^{2}, \quad \nabla_{\mu} \frac{1}{\nabla^{2}} \nabla_{\mu}=1, \\
& e^{-s \nabla^{2}} \nabla_{\mu} e^{s \nabla^{2}}=\left[\delta_{\mu \nu} \operatorname{ch}\left(4 \Lambda^{2} s\right)-i b_{\mu \nu} \operatorname{sh}\left(4 \Lambda_{\mathrm{g}}^{2} s\right)\right] \nabla_{\nu} \text {. }
\end{aligned}
$$

Пусть

$$
R_{\mu \nu}=-\nabla^{2} \delta_{\mu \nu}+i 4 \Lambda_{\mathrm{g}}^{2} b_{\mu \nu}, \quad R_{\mu \nu}^{-1}=\frac{-\nabla^{2} \delta_{\mu \nu}-i 4 \Lambda_{\mathrm{g}}^{2} b_{\mu \nu}}{\nabla^{4}-\left(4 \Lambda_{\mathrm{g}}^{2}\right)^{2}}
$$

тогда

$$
\left(\nabla R^{-1} \nabla\right)=\nabla_{\mu} R_{\mu \nu}^{-1} \nabla_{\nu}=1
$$




\section{Нулевые моды}

ПРИЛОЖЕНИЕ 4

Пусть оператор $R$, в нашем случае это

$$
R=-\square+\Lambda^{4} x^{2}-4 \Lambda^{2},
$$

имеет нулевые собственные значения, т.е.

$$
R U_{n}=\lambda_{n} U_{n}, \quad U_{n} \in L^{2}, \quad \lambda_{0}=0, \quad \lambda_{n}>0, \quad n>0 .
$$

Проблема состоит в том, как вычислить функциональный интеграл (20), в котором присутствуют нулевые моды в Гауссовской мере. Существует прямой способ устранения нулевых мод, изложенный в работе [10], где без всяких объяснений непосредственно в функциональный интеграл (20) вводится $\delta$-функция, выбрасываюшая нулевые моды из интеграла. Мы же хотим здесь изложить физические аргументы, оправдываюшие такое выбрасывание.

Итак, сформулируем проблему: найти функцию Грина, т.е. обратный оператор, которая в случае отсутствия нулевых мод $\left(\lambda_{n}>0, n \geqslant 0\right)$ может быть представлена как

$$
G=\frac{1}{R}=\sum_{n}\left|U_{n}\right\rangle \frac{1}{\lambda_{n}}\left\langle U_{n}|=| U_{0}\right\rangle \frac{1}{\lambda_{0}}\left\langle U_{0}\left|+\sum_{n>0}\right| U_{n}\right\rangle \frac{1}{\lambda_{n}}\left\langle U_{n}\right|
$$

Очевидно, это представление не имеет смысла в случае сушествования нулевых собственных значений $\lambda_{0}=0$.

Построение функции Грина, т.е. обратного оператора $G=1 / R$, эквивалентно решению следуюшей задачи: найти решение уравнения

$$
R \Phi=J
$$

с подходяшими краевыми условиями. В КТП требуется, чтобы решение было квадратично интегрируемо, т.е. $\Phi \in L^{2}$, если $J \in L^{2}$.

Уравнение (33) решим следуюшим образом. Поскольку собственные функции $\left\{U_{n}\right\}$ оператора $R$ образуют полную систему, разложим функцию $J$ в ряд

$$
J=\sum_{n=0}^{\infty} j_{n} U_{n},
$$

а функцию $\Phi$ будем искать в виде

$$
\Phi=j_{0} \phi_{0}+\sum_{n=1}^{\infty} c_{n} U_{n} .
$$


Подставив это разложение в уравнение (33), получим $c_{n}=j_{n} / \lambda_{n}$ для $n>0$ и уравнение на функцию $\phi_{0}$ :

$$
R \phi_{0}=U_{0} .
$$

Это уравнение имеет решение, следовательно, для функции Грина получим

$$
G=\frac{1}{R}=\left|\phi_{0}\right\rangle\left\langle U_{0}|+| U_{0}\right\rangle\left\langle\phi_{0}\left|+\sum_{n>0}\right| U_{n}\right\rangle \frac{1}{\lambda_{n}}\left\langle U_{n}\right|
$$

Эта функция Грина удовлетворяет уравнению

$$
R G=\left|U_{0}\right\rangle\left\langle U_{0}\left|+\sum_{n>0}\right| U_{n}\right\rangle\left\langle U_{n}\right|=I,
$$

т.е. действительно является функцией Грина оператора $R$, обладающего нулевыми собственными значениями.

Казалось бы, задача решена. Однако решение $\phi_{0}$ не квадратично-интегрируемо, т.е. $\phi_{0} \notin L^{2}$ ! В случае оператора (32)

$$
\phi_{0}(x) \sim e^{\Lambda^{2} x^{2} / 2}, \quad x^{2} \rightarrow \infty .
$$

Следовательно, решение не сушествует в пространстве $L^{2}$ и $\Phi=G J \notin L^{2}$. Если мы теперь встанем на стандартную точку зрения квантовой механики (только квадратично-интегрируемые функции имеют физический смысл), то мы должны поступить стандартным образом, т.е. исключить эти решения из допустимых функций, описываюших какое-либо физическое состояние.

Таким образом, в качестве функции Грина следует использовать

$$
G_{\mathrm{reg}}=\sum_{n>0}\left|U_{n}\right\rangle \frac{1}{\lambda_{n}}\left\langle U_{n}\right| \in L^{2} .
$$

Проше всего регуляризованную функцию Грина можно найти, воспользовавшись равенствами

$$
\begin{gathered}
G(z)=\frac{1}{R+z}, \quad G_{\mathrm{reg}}(z)=G(z)-\left.\frac{1}{z} \operatorname{Res}\right|_{\zeta=0} G(\zeta), \\
G_{\mathrm{reg}}=G_{\mathrm{reg}}(0)=\sum_{n>0}\left|U_{n}\right\rangle \frac{1}{\lambda_{n}}\left\langle U_{n}\right| \in L^{2} .
\end{gathered}
$$

Обратимся теперь к функциональному интегралу

$$
Z=\int D \Phi \exp \left(-\frac{1}{2}(\Phi R \Phi)+g W[\Phi]\right)=\exp \left(\frac{1}{2}\left(\frac{\delta}{\delta \Phi} G \frac{\delta}{\delta \Phi}\right)\right) e^{g W[\Phi]}=\sum_{n} \frac{g^{n}}{n !} Z_{n} .
$$


Слагаемые $Z_{n}$ не определены математически, поскольку при использовании функции Грина (34) интегралы, определяюшие $Z_{n}$, расходятся при больших $x$. Мы определим функциональный интеграл как

$$
Z \doteq \int D \Phi \exp \left(-\frac{1}{2}\left(\Phi R_{\mathrm{reg}} \Phi\right)+g W[\Phi]\right)=\exp \left(\frac{1}{2}\left(\frac{\delta}{\delta \Phi} G_{\mathrm{reg}} \frac{\delta}{\delta \Phi}\right)\right) e^{g W[\Phi]}
$$

где используется регуляризованная функция Грина (35).

Получим $G_{\mathrm{reg}}(x)$ для оператора $R(32)$, воспользовавшись формулами приложения $1:$

$$
\begin{aligned}
G_{\mathrm{reg}}(x)= & \left(\frac{1}{-\square+\Lambda^{4} x^{2}-4 \Lambda^{2}+z} \delta(x)\right)_{\mathrm{reg}}= \\
= & \frac{4 \Lambda^{4}}{(4 \pi)^{2}} \int_{0}^{\infty} d s \frac{2-e^{-4 \Lambda^{2} s}}{\operatorname{sh}^{2}\left(2 \Lambda^{2} s\right)} e^{-\Lambda^{2} x^{2} /\left(2 \operatorname{th}\left(2 \Lambda^{2} s\right)\right)}+ \\
& +\frac{8 \Lambda^{2}}{(4 \pi)^{2}} e^{-\Lambda^{2} x^{2} / 2} \int_{0}^{\infty} d t\left(e^{-\Lambda^{2} x^{2}(\operatorname{cth}(t)-1) / 2}-1\right) .
\end{aligned}
$$

В частности,

$$
G_{\mathrm{reg}}=G_{\mathrm{reg}}(0)=\frac{4 \Lambda^{4}}{(4 \pi)^{2}} \int_{s_{0}}^{\infty} d s \frac{2-e^{-4 \Lambda^{2} s}}{\operatorname{sh}^{2}\left(2 \Lambda^{2} s\right)}
$$

Сушественно, что при $\Lambda \rightarrow 0$ мы получаем правильный пропагатор безмассового скалярного поля:

$$
\lim _{\Lambda \rightarrow 0} G_{\text {reg }}(x)=\frac{1}{(4 \pi)^{2}} \int_{0}^{\infty} \frac{d s}{s^{2}} e^{-x^{2} / 4 s}=\frac{1}{(2 \pi)^{2} x^{2}}
$$

ПРИЛОЖЕНИЕ 5

\section{Кварковая энергия вакуума}

Вычислим кварковый вклад $E_{f}(\Lambda)$ в энергию вакуума. Имеем

$$
\begin{aligned}
E_{\mathrm{q}}(\Lambda) & =-\lim _{V \rightarrow \infty} \frac{1}{V} \operatorname{Tr} \ln \left[\frac{\gamma_{\mu} \breve{\nabla}_{\mu}(x)-M}{\gamma_{\mu} \partial_{\mu}-M}\right]= \\
& =-\lim _{V \rightarrow \infty} \frac{1}{2 V} \operatorname{Tr} \ln \left[\frac{-\left(\gamma_{\mu} \breve{\nabla}_{\mu}(x)\right)^{2}+M^{2}}{-\square+M^{2}}\right] .
\end{aligned}
$$

В слагаемом с производной $\gamma_{\mu} \breve{\nabla}_{\mu}(x)=\left(\gamma \partial_{x}\right)+i \Lambda^{2} \breve{n}(\gamma b x)$ цветовую матрицу $\check{n}$ выберем в диагональной форме, тогда и производная будет диагональна в цветовом пространстве:

$$
\gamma_{\mu} \breve{\nabla}_{\mu}(x)=\left(\gamma \partial_{x}\right)+i \breve{\Lambda}^{2}(\gamma b x)
$$

где

$$
\breve{\Lambda}^{2}=\Lambda^{2} \breve{n}=\operatorname{diag}\left(\frac{\Lambda^{2}}{2 \sqrt{3}}, \frac{\Lambda^{2}}{2 \sqrt{3}},-\frac{\Lambda^{2}}{\sqrt{3}}\right)
$$


Далее имеем

$$
\left(\gamma_{\mu} \breve{\nabla}_{\mu}(x)\right)^{2}=\nabla^{2}(y)-4 \breve{\Lambda}^{2} \mathcal{P}
$$

где матрица $\mathcal{P}$ совпадает с (30). Введем проекционные операторы (31) и разложим оператор (38) по этим проекционным операторам, тогда вакуумная энергия в выражении (37) для каждого отдельного кварка может быть представлена в форме

$$
\begin{aligned}
E_{\mathrm{q}}(\Lambda)= & -\lim _{V \rightarrow \infty} \frac{1}{V} \operatorname{Tr}\left\{\ln \left[\frac{-\square+\breve{\Lambda}^{4} y^{2}+M^{2}}{-\square+M^{2}}\right]+\right. \\
& \left.+\frac{1}{2} \ln \left[\frac{-\square+\breve{\Lambda}^{4} y^{2}+M^{2}-4 \breve{\Lambda}^{2}}{-\square+M^{2}}\right]+\frac{1}{2} \ln \left[\frac{-\square+\breve{\Lambda}^{4} y^{2}+M^{2}+4 \breve{\Lambda}^{2}}{-\square+M^{2}}\right]\right\} .
\end{aligned}
$$

Воспользовавшись формулами приложения 1, получим

$$
\begin{gathered}
E_{\mathrm{q}}(\Lambda)=4 \Lambda^{4}\left\{\frac{1}{(4 \pi)^{2}} W_{\mathrm{q}}\left(\frac{2 \Lambda^{2}}{M^{2}}\right)+\frac{2}{3} Z(M)\right\}, \\
W_{\mathrm{q}}(X)=\frac{1}{3}\left[W\left(\frac{X}{2 \sqrt{3}}\right)+2 W\left(\frac{X}{\sqrt{3}}\right)\right]= \begin{cases}\frac{1}{60} X^{2}+O\left(X^{3}\right), & x \rightarrow 0, \\
\frac{1}{3} \ln X+O(1), & x \rightarrow \infty .\end{cases}
\end{gathered}
$$

Функция $W(X)$ определена в $(27)$.

ПРИЛОЖКНИЕ 6

\section{Глюонная энергия вакуума}

Вычислим глюонную энергию вакуума. Рассмотрим функциональный интеграл (20). Прежде всего вычислим $\Delta(A)$. Согласно определению (см., например, [11])

$$
\Delta(A) \int d \omega \delta\left(\nabla A^{\omega}\right)=1
$$

В нашем приближении в низшем порядке теории возмушений $(g=0)$ имеем

$$
\Delta(A)=\operatorname{Tr} \ln \left(1-\frac{1}{\square} i \Lambda_{\mathrm{g}}^{2}\left(x_{\mu} b_{\mu \nu} \partial_{\nu}\right)\right)=1
$$

Матрища $\check{n}$ рассмотрена в приложении 2. Интегрирование в (20) по полям, соответствующим нулевым собственным значениям матрицы $\check{n}$, дает единицу. Поэтому ее можно рассматривать как $(4 \times 4)$-матрицу с собственными значениями $\pm \sqrt{3} / 4$, т.е.

$$
\Lambda^{2} \check{n} \Rightarrow \Lambda_{\mathrm{g}}^{2} \operatorname{diag}(1,1,-1,-1), \quad \Lambda_{\mathrm{g}}^{2}=\frac{\sqrt{3}}{4} \Lambda^{2}
$$

4 Теоретическая и математическая физика, т. 141, № 1, 2004 г. 
Функциональный интеграл для $\mathcal{Z}_{\mathrm{Vg}}^{(0)}$ в $(20)$ не зависит от знака тензора $b_{\mu \nu}$ и поэтому может быть представлен в форме произведения

$$
\begin{gathered}
\mathcal{Z}_{\mathrm{Vg}}^{(0)}=\left(\mathcal{Y}_{\mathrm{V}}\right)^{4}, \\
\mathcal{Y}_{\mathrm{V}}=\frac{1}{N_{A}} \int D A \delta\left(\nabla_{\mu} A_{\mu}\right) \exp \left(-\frac{1}{2} \int_{\mathrm{V}} d x\left(A_{\mu} R_{\mu \nu} A_{\nu}\right)\right), \\
R_{\mu \nu}=-\nabla^{2} \delta_{\mu \nu}+4 i \Lambda_{\mathrm{g}}^{2} b_{\mu \nu}, \quad \nabla_{\mu}(x)=\partial_{\mu}+i \Lambda_{\mathrm{g}}^{2} b_{\mu \nu} x_{\nu} .
\end{gathered}
$$

Постоянная $N_{A}$ определена условием $\left.\mathcal{Y}_{\mathrm{V}}\right|_{\Lambda=0}=1$. Вычислим интеграл

$$
\begin{aligned}
\mathcal{Y}_{\mathrm{V}} & =\frac{1}{N_{A}} \int D A \delta(\nabla A) e^{-(A R A) / 2}=\frac{1}{N_{A}} \int D S \int D A e^{-(A R A) / 2+i(S \nabla A)}= \\
& =\left(\frac{\operatorname{Det} R_{\mu \nu}^{0}}{\operatorname{Det} R_{\mu \nu}}\right)^{1 / 2} \int D S e^{-\left(S \nabla R^{-1} \nabla S\right) / 2}=\left(\frac{\operatorname{Det} R_{\mu \nu}^{0}}{\operatorname{Det} R_{\mu \nu}} \frac{1}{\operatorname{Det}\left(\nabla R^{-1} \nabla\right)}\right)^{1 / 2}= \\
& =\operatorname{Det}\left[\frac{-\square}{-\nabla^{2}+4 \Lambda_{\mathrm{g}}^{2}}\right] \operatorname{Det}\left[\frac{-\square}{-\nabla^{2}-4 \Lambda_{\mathrm{g}}^{2}}\right] .
\end{aligned}
$$

Таким образом, для глюонного вклада в энергию вакуума получаем представление

$$
E_{\mathrm{g}}(\Lambda)=4 \lim _{V \rightarrow \infty} \frac{1}{V} \operatorname{Tr}\left\{\ln \left[\frac{-\square+\Lambda_{\mathrm{g}}^{4} x^{2}+4 \Lambda_{\mathrm{g}}^{2}}{-\square}\right]+\ln \left[\frac{-\square+\Lambda_{\mathrm{g}}^{4} x^{2}-4 \Lambda_{\mathrm{g}}^{2}}{-\square}\right]\right\} .
$$

Оператор $-\square+\Lambda_{\mathrm{g}}^{4} x^{2}-4 \Lambda_{\mathrm{g}}^{2}$ содержит нулевые собственные значения. Обсуждение проблемы нулевых мод дано в приложении 4 , согласно которому имеем

$$
\begin{aligned}
E_{\mathrm{g}}\left(\Lambda_{\mathrm{g}}\right) & =8 \frac{4 \Lambda_{\mathrm{g}}^{4}}{(4 \pi)^{2}} \int_{s_{0}}^{\infty} \frac{d s}{s}\left[\frac{1}{\left(2 \Lambda_{\mathrm{g}}^{2} s\right)^{2}}-\frac{\operatorname{ch}\left(4 \Lambda_{\mathrm{g}}^{2} s\right)}{\operatorname{sh}^{2}\left(2 \Lambda_{\mathrm{g}}^{2} s\right)}+2\right]= \\
& =\frac{3}{2} \frac{4 \Lambda^{4}}{(4 \pi)^{2}} \int_{s_{0}}^{\infty} \frac{d s}{s} \phi\left(2 \Lambda_{\mathrm{g}}^{2} s\right),
\end{aligned}
$$

где

$$
\phi(t)=\frac{1}{t^{2}}-\frac{1}{\operatorname{sh}^{2} t} .
$$

Выделим расходяшуюся часть из интеграла:

$$
\begin{aligned}
\int_{s_{0}}^{\infty} \frac{d s}{s} \phi\left(2 \Lambda_{\mathrm{g}}^{2} s\right) & =\int_{s_{0}}^{a} \frac{d s}{s} \phi(0)+\int_{s_{0}}^{a} \frac{d s}{s}\left[\phi\left(2 \Lambda_{\mathrm{g}}^{2} s\right)-\phi(0)\right]+\int_{a}^{\infty} \frac{d s}{s} \phi\left(2 \Lambda_{\mathrm{g}}^{2} s\right) \Rightarrow \\
& \Rightarrow \frac{1}{3} \ln \left(\frac{a}{s_{0}}\right)+\int_{0}^{2 \Lambda_{\mathrm{g}}^{2} a} \frac{d t}{t}[\phi(t)-\phi(0)]+\int_{2 \Lambda_{\mathrm{g}}^{2} a}^{\infty} \frac{d t}{t} \phi(t)= \\
& =\frac{1}{3} \ln \left(\frac{H^{2}}{2 s_{0}}\right)-\frac{1}{3} \ln \left(\frac{2 \Lambda^{2}}{H^{2}}\right), \quad a=\frac{1}{H^{2}} .
\end{aligned}
$$

Поскольку в глюонном лагранжиане отсутствует какая-либо шкала длин, здесь возникает неопределенный параметр $H$, который должен быть связан с хромодинамической энергетической шкалой $\Lambda_{\mathrm{Kх}}$ д 
Окончательно глюонный вклад в энергию вакуума записывается в виде

$$
E_{\mathrm{g}}(\Lambda)=-\frac{4 \Lambda^{4}}{(4 \pi)^{2}} \frac{1}{2} \ln \left(\frac{2 \Lambda^{2}}{H^{2}}\right)
$$

где слагаемое, содержашее $s_{0}$, удалено соответствуюшей перенормировкой.

Благодарности. Работа поддержана грантом РФФИ № 04-02-17370.

\section{Список литературы}

[1] E. S. Swan. Aspects of confinement: a brief review. hep-ph/0310089.

[2] P. Maris, G. D. Roberts. Int. J. Mod. Phys. E. 2003. V. 12. P. 297; nucl-th/0301049.

[3] G. V. Efimov, G. Ganbold. Phys. Rev. D. 2002. V. 65. P. 054012.

[4] G. V. Efimov, S. N. Nedelko. Phys. Rev. D. 1995. V. 51. P. 176; Eur. Phys. J. C. 1998. V. 1. P. 343.

[5] Ya. V. Burdanov, G. V. Efimov. Phys. Rev. D. 2001. V. 64. P. 014001.

[6] G. K. Savvidy. Phys. Lett. B. 1977. V. 71. P. 133.

[7] H. Leutwyler. Nucl. Phys. B. 1981. V. 179. P. 129.

[8] A. С. Вайтман. Проблемы в релятивистской динамике квантованных полей. М.: Наука, 1968.

[9] J. Schwinger. Phys. Rev. 1951. V. 82. P. 664.

[10] D. I. Dyakonov, V. Yu. Petrov. Nucl. Phys. B. 1984. V. 245. P. 269.

[11] А. А. Славнов, Л. Д. Фаддеев. Введение в квантовую теорию калибровочных полей. М.: Наука, 1988.

\footnotetext{
Поступила в редакцию 13.I.2004 г.,
} после доработки 24.III.2004 г. 